\title{
Exoplanet Atmospheres and Photochemistry
}

\author{
S. Seager ${ }^{1}$, M.-C. Liang ${ }^{2}$, C. D. Parkinson ${ }^{2}$, and Y. L. Yung ${ }^{2}$ \\ ${ }^{1}$ Department of Terrestrial Magnetism, Carnegie Institution of Washington, \\ 5241 Broad Branch Rd. NW, Washington, DC 20015, USA \\ ${ }^{2}$ Division of Geological and Planetary Sciences, California Institute of Technology, \\ 1201 East California Boulevard, Pasadena, CA 91125, USA
}

\begin{abstract}
Over 150 extrasolar planets are known to orbit sun-like stars. A growing number of them (9 to date) are transiting "hot Jupiters" whose physical characteristics can be measured. Atmospheres of two of these planets have already been detected. We summarize the atmosphere detections and useful upper limits, focusing on the MOST albedo upper limit and $\mathrm{H}$ exosphere detection for HD 209458b as the most relevant for photochemical models. We describe our photochemical model for hot Jupiters and present a summary explanation of the main results: a low gas-phase abundance of hydrocarbons; an absence of hydrocarbon hazes; and a large reservoir of $\mathrm{H}$ atoms in the upper atmospheres of hot Jupiters. We conclude by relating these model results to the relevant observational data.
\end{abstract}

Keywords. planetary systems — radiative transfer

\section{Introduction}

Over 150 extrasolar planets are now known to orbit main-sequence stars. Most of the extrasolar planets have been discovered by the Doppler technique (Marcy et al. 2005; Santos et al. 2005), which measures the star's motion about the planet-star common center of mass. The resulting minimum masses and orbital parameters are guiding planet formation and migration models. The planet transit survey technique has recently succeeded in discovering exoplanets. Although planets are known to orbit pulsars and direct imaging of young stellar systems has revealed bright, low-mass objects 100s of AU from their star, here we focus on solar-system-aged planets around main-sequence stars.

Now that the extrasolar planets' existence is firmly established, we want to learn more about their physical properties. Direct imaging of solar-system-aged planets is not yet possible, because the adjacent star outshines the planet by up to ten orders of magnitude. Fortunately, transiting planets provide us with many opportunities for observations with current technology. In particular, the transiting hot Jupiter (planets within $0.05 \mathrm{AU}$ of their stars) planet atmospheres have been successfully detected. The hot Jupiter HD 209458b has been extensively observed because, until recently, it was the only one hosted by a bright star.

\section{Transiting Planet Data}

\subsection{Primary Transit}

When an exoplanet goes in front of its parent star, both its density and atmosphere can potentially be measured. The drop in brightness of a parent star during transit gives the planet-to-star area. If the parent star's radius is known (usually known to about 10\% for main-sequence stars), so is the planet's. A mass measurement is needed to identify a transiting object as a planet, because low-mass stars, brown dwarfs (i.e., failed stars) 
and giant planets all have similar sizes due to the hydrogen equation of state. The mass and radius give planetary density.

During planet transit some of the starlight passes through part of the planet's atmosphere. The planet's "transmission spectrum", therefore, is hiding in the stellar spectrum during transit. Astronomers can compare the stellar spectrum before and during transit to get the planet's spectrum. Detecting the transmission spectrum is a very difficult measurement, because the planet's spectrum is on order 10,000 times fainter than the star's. The differential nature of the measurement and the on/off nature of the transit is what enables such a precise measurement. For the planet HD 209458b, the following useful observations or upper limits have been obtained:

- $\mathrm{Na}$ atmosphere detection (Charbonneau et al. 2002);

- H Ly $\alpha$ exosphere detection (Vidal-Madjar et al. 2003);

- CO atmosphere upper limit (Deming et al. 2005a).

The H Ly $\alpha$ detection is the transmission spectrum observation most relevant to photochemistry. A huge occulting area in Ly $\alpha$ was detected, a 15\% drop in stellar brightness. This is a 10 times greater area than the planet's transit at visible wavelengths - implying an extended exosphere out to 3 or 4 Jupiter radii. Theoretical estimates for hydrogen escape from hot Jupiter atmospheres (Baraffe et al. 2004; Yelle 2004; Hubbar et al. 2005) vary over 4 orders of magnitude - translating from minimal to substantial planet mass loss over the planet's lifetime. Researchers do agree that an extremely high thermospheric temperature of $10,000 \mathrm{~K}$ is required for atmospheric escape to explain the Vidal-Madjar et al. (2003) observations.

\subsection{Secondary Eclipse}

A planet that passes in front of its star also goes behind its star. A differential measurement before and after the planet enters into "secondary eclipse" can be used to measure the thermal emission from the planet. The thermal emission measurement is easier than the transmission spectrum measurement, because the whole face of the planet contributes to the signal and not just the thin shell of the atmosphere. The detections or useful upper limits for HD 209458b and where noted for the planet TrES-1 are:

- Thermal emission at 24 microns (Deming et al. 2005);

- Thermal emission TrES-1 at 4.5 and 8 microns (Charbonneau et al. 2005);

- $\mathrm{H}_{2} \mathrm{O}$ upper limit 2.2 microns (Richardson et al. 2003b);

- Albedo upper limit, 0.3-0.7 microns (Rowe et al. 2005).

The albedo upper limit is the secondary eclipse observation most relevant to photochemistry. This was measured with the Canadian Space Agency microsatellite called MOST (Microvariability and Oscillations of STars; Walker et al. 2003; Matthews et al. 2004). The upper limit on the geometric albedo is $0.25(1 \sigma)$ in MOST's single broad-band filter which spans $0.3-0.7$ microns. With an albedo of 0.5 in the same bandpass (using data from Karkoschka 1994), Jupiter is much more reflective than HD 209458b.

\section{Model Atmospheres and Chemical Equilibrium}

Model atmospheres used for extrasolar giant planets are typically 1D, plane-parallel, LTE (local thermodynamic equilibrium), hydrostatic equilibrium and chemical equilibrium computer models (see Marley et al. 2005; Seager et al. 2005 and references therein). Hot Jupiters may range in equilibrium temperature from 900-1800 K depending on albedo. The major uncertainty for the model is clouds: their composition, particle size distribution, fraction of gas condensed and cloud vertical extent. The clouds would be composed of high-temperature condensates, including potentially silicates and iron. The 
second most important uncertainty is heat redistribution by atmospheric circulation. The hot Jupiters are almost certainly "tidally-locked", presenting the same face to the star at all times (just as our moon does to Earth). In an orbit almost 10 times closer to their stars as Mercury is to our sun, these planets are intensely heated. Strong winds, even approaching the sound speed, may or may not efficiently redistribute the energy (e.g., Showman \& Guillot 2002; Cho et al. 2003; Cooper \& Showman 2005). Other model uncertainties include opacities, internal luminosities, and non-equilibrium chemistry.

Considering chemical equilibrium we can specify the major chemical species in a hot Jupiter atmosphere. Figure 1 shows some of the abundant chemical species (expressed as partial pressure) for solar abundances present at 0.1 bar for different temperatures. This pressure/temperature regime can be thought of as representative of the atmospheric location where the spectral lines form. Although most hydrogen is in the form of $\mathrm{H}_{2}$, $\mathrm{H}_{2} \mathrm{O}$ is abundant. $\mathrm{H}_{2} \mathrm{O}$ is a strong absorber and its absorption features are expected to sculpt the near-IR spectrum of hot Jupiters (Figure 2). For solar abundances CO is the dominant form of carbon at high temperatures, whereas $\mathrm{CH}_{4}$ dominates at lower temperatures. This makes the potential detection of $\mathrm{CO}$ or $\mathrm{CH}_{4}$ a useful temperature discriminant. The hot Jupiter atmospheres also "live" in the regime of high-temperature condensates. Two examples are shown in Figure 1: Fe vapor converting into solid Fe and Ti becoming locked into Ti condensates at low temperature. The latter is significant, since $\mathrm{TiO}$ is the most important absorber for low-mass (i.e., M) stars at visible wavelengths, and hence likely will not be present in the cooler hot Jupiter atmospheres. The alkali metals are expected to be in their atomic form in hot Jupiter atmospheres, due to their low ionization potentials. This is in contrast to both stars, where most of a given alkali metal is ionized, and to Jupiter where alkali metals are locked into condensates deep in the atmosphere. See the excellent review by Lodders (2005) for details on chemistry in brown dwarf atmospheres - this also applies to hot Jupiter atmospheres which are similar in temperature to brown dwarf atmospheres.

Fortney et al. (2005) and Seager et al. (2005) have made forays into hot Jupiter atmosphere models with non-solar abundances, in particular exploring the $\mathrm{C} / \mathrm{O}$ ratio. With a higher $\mathrm{C}$ ratio, $\mathrm{CO}$ becomes more abundant at the expense of $\mathrm{H}_{2} \mathrm{O} \cdot \mathrm{CH}_{4}$ also becomes more abundant than in the solar abundance case.

Given the chemical equilibrium profile, we can understand the "big picture" spectrum of a hot Jupiter. Figure 2 shows a theoretical spectrum for a typical hot Jupiter (in this case the $51 \mathrm{Peg}$ system). The stellar spectrum is computed from Kurucz model atmospheres (Kurucz 1993). In this theoretical example, the planet's visible wavelength spectrum is dominated by scattered light, showing the same features as in the star. At visible wavelengths the alkali metals $\mathrm{Na}$ and $\mathrm{K}$ are strong absorbers, but in the IR strong $\mathrm{H}_{2} \mathrm{O}$ absorption features and some $\mathrm{CH}_{4}$ bands shape the spectrum. See Seager et al. (2000, 2005) for details.

\section{Photochemistry}

The spectra of solar system giant planets Jupiter and Saturn are affected by photochemistry. Solar UV radiation photodissociates $\mathrm{CH}_{4}$, and the subsequent reactions with photolytic products of $\mathrm{CH}_{4}$ synthesize higher hydrocarbons, in turn forming hydrocarbon hazes. The hazes make the UV- and blue-wavelength albedos lower by a factor of 2 to 3 and "wash out" spectral features. See Figure 3 in Karkoschka (1994) for full disk albedo spectra of Jupiter, Saturn, Uranus, and Neptune for the effects of photochemistry.

Hot Jupiters are 100 times closer to their star than Jupiter is to the Sun and therefore receive $10^{4}$ times as much flux. This raises the question about whether hydrocarbon hazes 


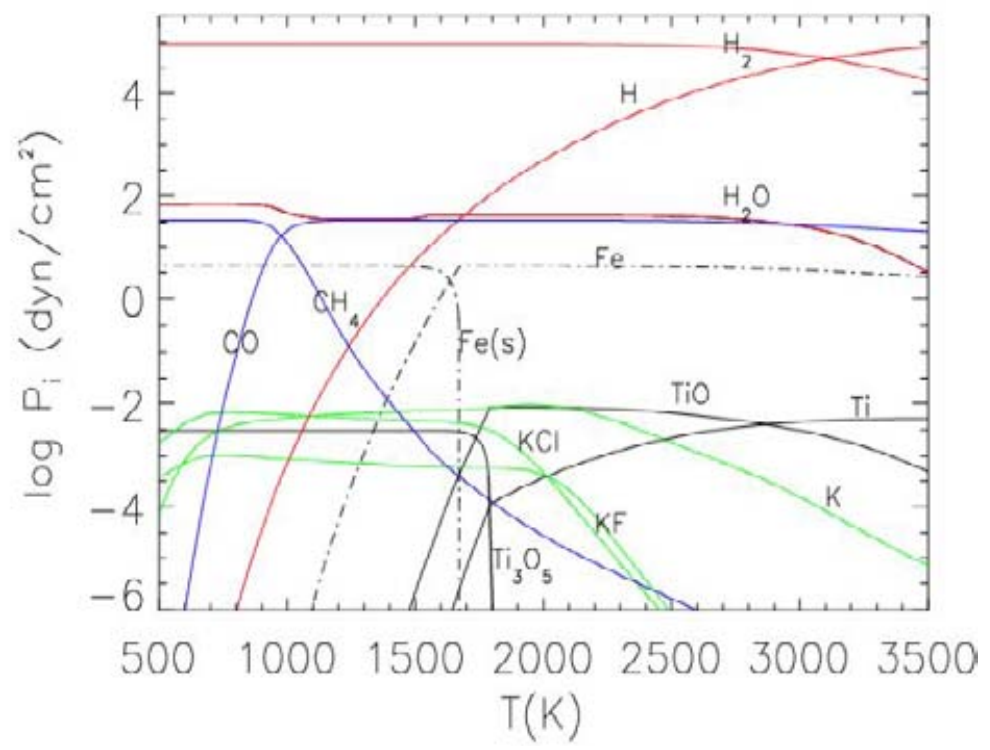

Figure 1. Partial pressures of some molecules and solids in a typical hot Jupiter atmosphere. Solar abundances are used. The total pressure is $10^{5}$ dyne $\mathrm{cm}^{-2}(0.1 \mathrm{bar})$, representative of where spectral lines are formed. A Gibbs free energy minimization routine was used for this computation, described in Seager (1999).

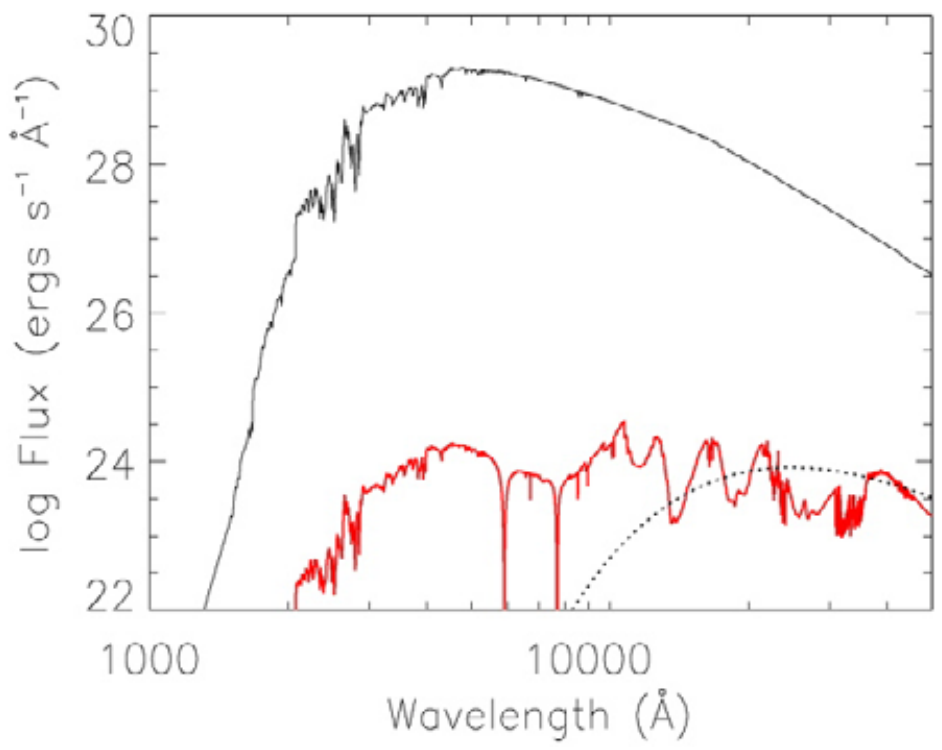

Figure 2. Generic spectrum of a hot Jupiter planet and its parent star. See text for details.

are highly abundant and in turn cause an even more extreme affect on the planetary visible-wavelength spectra.

We have modeled photochemistry on hot Jupiters using the 1D Caltech/JPL KINETICS model developed by Yuk Yung's group (Strobel 1973; Gladstone et al. 1996). The model reproduces hydrocarbon observations on Jupiter, as well as He $584 \AA$ and H Ly $\alpha$ 


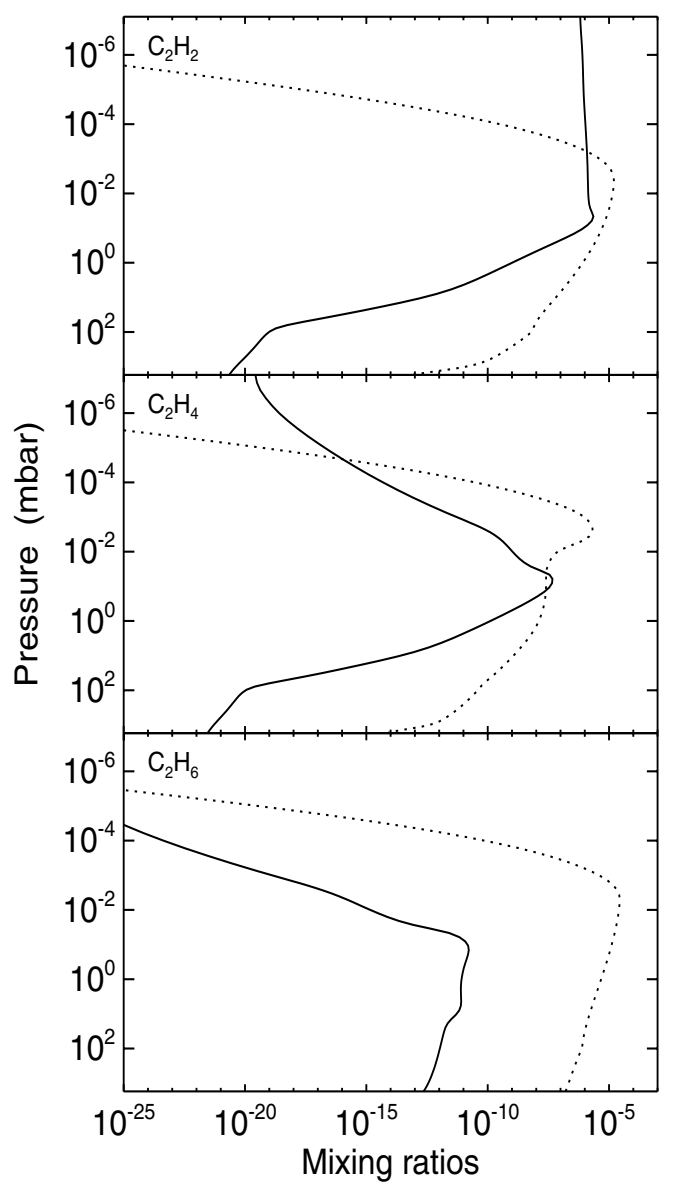

Figure 3. Mixing ratios of photochemical products in hot Jupiter atmospheres. The solid line is for a fiducial hot Jupiter atmosphere model (see Liang et al. 2004) and the dotted line is for Jupiter. Overall, the hot Jupiter model shows a low total gas-phase abundance of hydrocarbons. These three hydrocarbon compounds are basic ingredients for synthesizing complex hydrocarbons, such as benzene and PAHs, which are also expected to be of low abundance.

airglow on Jupiter. We used a reduced version involving four parent molecules: $\mathrm{H}_{2}, \mathrm{CO}$, $\mathrm{H}_{2} \mathrm{O}$, and $\mathrm{CH}_{4}$, and 253 chemical reactions.

The hot Jupiter atmosphere composition and temperature/pressure profiles are unknown. To overcome these uncertainties, we used three different input atmospheres (i.e., temperature/pressure profiles) from Seager et al. (2000), Barman et al. (2002), Fortney et al. (2003). We also used five different initial values of the input species, $\mathrm{H}_{2}, \mathrm{CO}, \mathrm{H}_{2} \mathrm{O}$ and $\mathrm{CH}_{4}$, and considered a four orders of magnitude variation over the $\mathrm{CH}_{4}$ abundance. The model and results are fully described in Liang et al. (2003, 2004). See those papers for a quantitative description of results.

The main results are as follows. No hydrocarbon hazes are expected on hot Jupiters and gas-phase hydrocarbons should have a very low abundance (Figure 3). These results are due to the hot Jupiters' proximity to their parent stars.

Primarily, the hot temperatures create fundamentally different chemical pathways than on Jupiter (Figure 4). Although the hydrocarbon formation rate is higher than on Jupiter, 


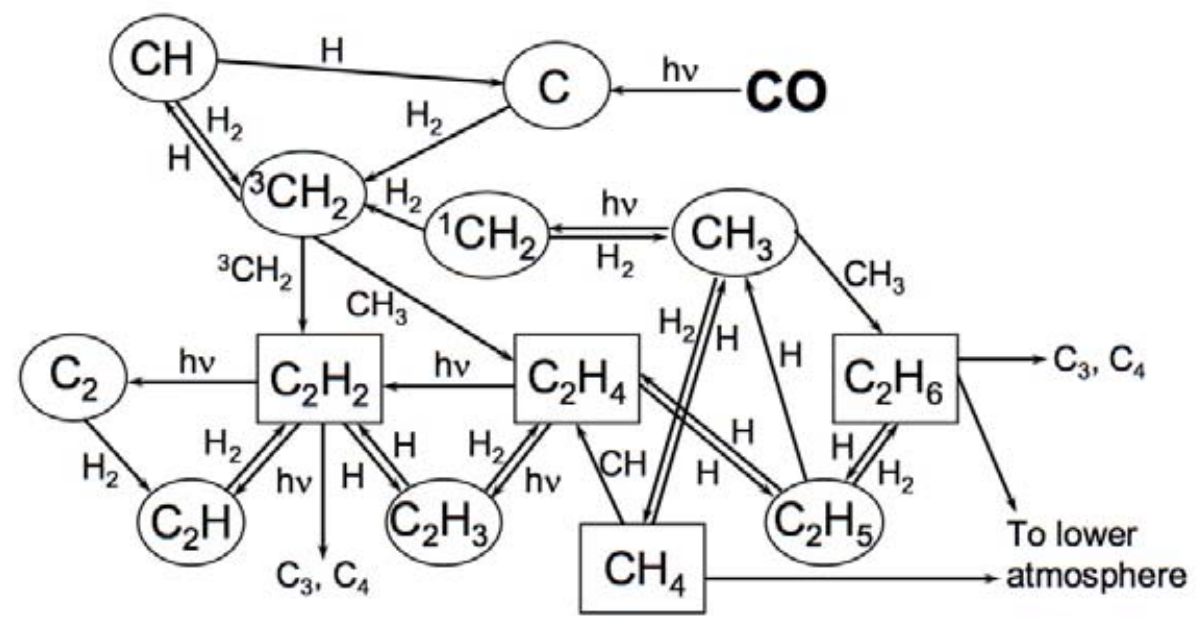

Figure 4. Photochemical pathways in hot Jupiter atmospheres.

so is the hydrocarbon destruction rate (i.e., the lifetime is decreased). This is because a key reaction in the destruction of $\mathrm{C}_{2} \mathrm{H}_{2}$ is fast at the high temperatures found on hot Jupiters, but a bottleneck at the low temperature found on Jupiter. The reaction is $\left(\mathrm{C}_{2} \mathrm{H}_{3}+\mathrm{H}_{2} \rightarrow \mathrm{C}_{2} \mathrm{H}_{4}+\mathrm{H}\right)$, and see Liang et al. (2003) for the full set of reactions. Because $\mathrm{C}_{2} \mathrm{H}_{2}$ is the main starting point for higher hydrocarbons, its low abundance leads to an overall low hydrocarbon abundance on hot Jupiters.

Secondly, $\mathrm{CO}$ and $\mathrm{H}_{2} \mathrm{O}$ are expected to be abundant on hot Jupiters at the expense of $\mathrm{CH}_{4}$, which is the dominant form of $\mathrm{C}$ on the colder solar system giant planets. Hydrocarbon formation on Jupiter is driven by the photodissociation of $\mathrm{CH}_{4}$ and the subsequent reactions of the products $-\mathrm{C}_{2} \mathrm{H}_{2}, \mathrm{C}_{2} \mathrm{H}_{4}, \mathrm{C}_{2} \mathrm{H}_{6}$ - are important for forming more complex hydrocarbons and hydrocarbon aerosols. The high-UV radiation environment close to the star, together with abundant $\mathrm{H}_{2} \mathrm{O}$, facilitates hydrocarbon destruction. $\mathrm{H}_{2} \mathrm{O}$ photodissociation in the upper atmosphere provides excess atomic $\mathrm{H}$ that partially enables the fast destruction of $\mathrm{C}_{2} \mathrm{H}_{2}$ by hydrogenation to $\mathrm{CH}_{4}$ (with the second criteria being temperature, described above) and forms a large atomic reservoir in the upper atmosphere. This is in contrast to the equilibrium chemistry case where all $\mathrm{H}$ exists as $\mathrm{H}_{2}$.

These results tie in to observations of HD 209458b described in $\S 2$. First, the low albedo on HD 209458b is not caused by hydrocarbon hazes. Second, the extended hydrogen exosphere can form partially because of a huge $\mathrm{H}$ reservoir in the planet's upper atmosphere, caused by $\mathrm{H}_{2} \mathrm{O}$ photodissociation.

\section{Near Future Prospects}

In the coming year, the Spitzer Space telescope will observe four transiting hot Jupiters to detect their thermal emission during secondary eclipse. One of these planets, HD 209458b, also has a growing dataset of observations and upper limits at different wavelengths and a good understanding of this planet's atmosphere should emerge. See Seager et al. (2005) for a discussion about future prospects. The number of transiting hot Jupiters around bright stars is growing and an increasing number will be characterized in terms of atmospheric composition, temperature and presence of an exosphere. On the 
computational side, photochemistry of $\mathrm{S}$ and $\mathrm{N}$ compounds needs to be further explored in their role as hazes on hot Jupiter atmospheres. The near future is bright for both observations and models of transiting hot Jupiters.

\section{Acknowledgements}

S.S. thank the conference organizers for an interesting meeting.

\section{References}

Baraffe, I., Selsis, F., Chabrier, G., Barman, T.S., Allard, F., Hauschildt, P.H., \& Lammer, H. 2004, A\&3A 419, L13

Barman, T.S., Hauschildt, P.H., Schweitzer, A., Stancil, P.C., Baron, E., \& Allard, F. 2002, Ap. J. 569, L51

Charbonneau, D., Brown, T.M., Noyes, R.W., \& Gilliland, R.L. 2002, Ap. J. 568, 377

Charbonneau, D., et al. 2005, Ap. J. 626, 523

Cho, J.Y.-K., Menou, K., Hansen, B.M.S., \& Seager, S. 2003, Ap. J. 587, L117

Cooper, C.S. \& Showman, A.P. 2005, Ap. J. 629, L45

Deming, D., Brown, T.M., Charbonneau, D., Harrington, J., \& Richardson, L.J. 2005, Ap. J. 622,1149

Deming, D., Seager, S., Richardson, L.J., \& Harrington, J. 2005, Nature 434, 740

Fortney, J.J., Marley, M.S., Lodders, K., Saumon, D., \& Freedman, R. 2005, Ap. J. 627, L69

Fortney, J.J., Sudarsky, D., Hubeny, I., Cooper, C.S., Hubbard, W.B., Burrows, A., \& Lunine, J.I. 2003, Ap. J. 589, 615

Gladstone, G.R., Allen, M., \& Yung, Y.L. 1996, Icarus 119, 1

Hubbard . W.B., Hattori M.F., Burrows, A., Hubeny, I., \& Sudarsky, D. Icarus, submitted

Karkoschka, E. 1994, Icarus 111, 174

Kurucz, R.L. 1993, VizieR Online Data Catalog, 6039, 0

Liang, M.-C., Seager, S., Parkinson, C.D., Lee, A.Y.-T., \& Yung, Y.L. 2004, Ap. J. 605, L61

Liang, M.-C., Parkinson, C.D., Lee, A.Y.-T., Yung, Y.L., \& Seager, S. 2003, Ap. J. 596, L247

Lodders, K. 2005, Science, in press

Marcy, G, Butler, R.P., Fischer, D., Vogt, S., Wright, J.T., Tinney, C.G., \& Jones, H. 2005, Prog. Theor. Phys Supp., in press

Marley, M.S., Fortney, J.J., Seager, S., \& Barman, T. 2005, PPV Conference Proceedings, in press

Matthews, J.M., Kusching, R., Guenther, D.B., Walker, G.A.H., Moffat, A.F.J., Rucinski, S.M., Sasselov, D., \& Weiss, W.W. 2004, Nature 430, 51

Rowe, J. et al. 2005, Ap. J., submitted

Richardson, L.J., Deming, D., \& Seager, S. 2003, Ap. J. 597, 581

Richardson, L.J., Deming, D., Wiedemann, G., Goukenleuque, C., Steyert, D., Harrington, J., \& Esposito, L.W. 2003, Ap. J. 584, 1053

Santos, N.C., Benz, W., \& Mayor, M. 2005, Science 310, 251

Seager, S., Richardson, L.J., Hansen, B.M.S., Menou, K., Cho, J.Y.-K., \& Deming, D. 2005, Ap. J. 632,1122

Seager, S., Whitney, B.A., \& Sasselov, D.D. 2000, Ap. J. 540, 504

Seager, S. 1999, Ph.D. Thesis,

Shkolnik, E., Walker, G.A.H., Bohlender, D.A., Gu, P.-G., \& Kuumlrster, M. 2005, Ap. J. 622, 1075

Showman, A.P. \& Guillot, T. 2002, A\&A 385, 166

Strobel, D.F. 1973, Journal of Atmospheric Sciences 30, 489

Vidal-Madjar, A., et al. 2004, Ap. J. 604, L69

Vidal-Madjar, A., Lecavelier des Etangs, A., Désert, J.-M., Ballester, G.E., Ferlet, R., Hébrard, G., \& Mayor, M. 2003, Nature 422, 143

Walker, G., et al. 2003, PASP 115, 1023

Yelle, R.V. 2004, Icarus 170, 167 


\section{Discussion}

BLACK: What can be said about magnetospheres and ionospheres of hot exoplanets?

SEAGER: We do not know if the hot exoplanets have magnetospheres. If they do, interaction between the stellar and planetary magnetic fields could show up observationally, because of their close proximity. Shkolnik et al. (2005) find suggestive evidence that two stars hosting hot Jupiters have chromospheric activity with the same period as the planet, suggesting a magnetic field interaction. No dramatic superflares from magnetic reconnection have been observed. The decametric radiation (as seen from Jupiter) due to synchrotron emission, is too faint to be detected with today's radio telescopes.

HerBst: Are your "equilibrium" chemical models truly thermodynamic ones? If so, they are probably used to too low temperatures.

SEAGER: The JANAF Thermochemical tables go down to 500K or much lower, so the calculations are appropriate.

VAN DISHOECK: What are the prospects for detecting the other $\mathrm{H}_{2} \mathrm{O}$ photodissociation products, $\mathrm{OH}$ or $\mathrm{O}$ ?

SEAGER: With the demise of HST/STIS we do not have any way of detecting narrow absorption features in the UV-visible wavelengths at this time. In addition, because the transmission spectrum measurement is so difficult, an atomic or molecular feature in an exoplanet atmosphere has to be extremely strong. However, a tentative detection of $\mathrm{O}$ in HD 209458b's exosphere has been reported by Vidal-Madjar et al. (2004).

StRELNITSKI: We have calculated the possibility of fluorescence and maser radiation in radio lines of molecules $\left(\mathrm{H}_{2} \mathrm{O}\right.$ in particular) from exoplanets. An important parameter for possible masering is the pressure of the gas. What is the typical pressure in the region where $\mathrm{H}_{2} \mathrm{O}$ is abundant?

SEAGER: For solar abundance models we expect water vapor to be abundant throughout the planet atmosphere - this includes pressures ranging from 10 bars to $\sim 10^{-5}$ bars, and possibly even a greater range. 